Review

\title{
Development of Macozinone for TB treatment: An Update
}

\author{
Vadim Makarov ${ }^{1, *}$ and Katarína Mikušová ${ }^{2}$ \\ 1 Federal Research Center "Fundamentals of Biotechnology RAS", Leninsky prospect 33, bld. 2, \\ 119071 Moscow, Russia \\ 2 Department of Biochemistry, Faculty of Natural Sciences, Comenius University in Bratislava, \\ Mlynská dolina, Ilkovičova 6, 84215 Bratislava, Slovakia; katarina.mikusova@uniba.sk \\ * Correspondence: makarov@inbi.ras.ru
}

Received: 5 March 2020; Accepted: 25 March 2020; Published: 26 March 2020

check for updates

\begin{abstract}
Macozinone, a piperazine-benzothiazinone PBTZ169, is currently undergoing Phase 1/2 clinical studies for the treatment of tuberculosis (TB). In this review we summarize the key findings that led to the development of this compound and to identification of its target, decaprenylphospohoryl ribose oxidase DprE1, which is involved in the synthesis of the essential arabinan polymers of the cell wall in a TB pathogen, Mycobacterium tuberculosis. We present the results of the pilot clinical studies, which raise optimism regarding its further development towards more efficient TB drug regimens.
\end{abstract}

Keywords: macozinone; DprE1 inhibitor; clinical studies

\section{Introduction}

Tuberculosis (TB) as both an acute and chronic infectious disease is still unbeaten and represents an important social, medical, and biological challenge for the healthcare worldwide [1]. The infection caused by Mycobacterium tuberculosis strains is most often located in the respiratory tract from which it is transmitted in the population by aerosols. At the same time, mycobacteria can be disseminated also to other organs. However, it is now well acknowledged that even in its primary location, the TB pathogen is present in different microenvironments and thus in various metabolic states, which substantially complicates the treatment [2]. According to the last Global Tuberculosis Report issued by the World Health Organization (WHO) [3], in 2018 the death rate was estimated to be about 1.45 million people. Among the estimated 10 million new cases of TB, 3.4\% had multidrug-resistant TB or rifampicin-resistant TB (MDR/RR-TB). This number increased to $18 \%$ in previously treated cases, with the highest proportions ( $>50 \%$ in previously treated cases) in countries of the former Soviet Union. Although efficient TB treatment is available, only 7 million new cases were treated for the disease in 2018, while an estimated 3 million patients did not have access to quality care or were not reported, and only one in three people with drug-resistant TB accessed care [3].

In addition to interventions in the social area and point-of-care management of $\mathrm{TB}$, one of the key challenges in making the fight against TB more effective is to improve treatment options. The current chemotherapy for the drug-sensitive TB takes about six months and requires administration of four different medicines (isoniazid, rifampicin, ethambutol, pyrazinamide) to avoid development of drug resistance. The length of the treatment and its adverse effects result in bad compliance and thus it is imperative to find novel drugs and regimens that would be less demanding on patients [4].

During recent years, three novel drugs against TB were approved. These are bedaquiline [5], delamanid [6] and pretomanid [7], which are currently undergoing several clinical trials aimed at treatment of the drug-resistant TB, as well as at shortening standard TB treatment [8]. The efforts and hope for better TB drugs are exemplified also by the pipeline of several new molecules, that 
are currently at different stages of clinical development. Among them there are inhibitors of protein synthesis from the oxazolidinone family (delpazolid, sutezolid, contezolid, TBI-223), inhibitor of leucyl-tRNA synthetase (GSK3036656), inhibitor of DNA gyrase B (SPR720), compounds interfering with the respiratory chain of a TB pathogen (telacebec, TBI-166), a cell wall inhibitor targeting MmpL3 transporter (SQ109) and four compounds targeting another cell wall target, DprE1. These are BTZ043, TBA-7371, OPC-167832, and macozinone (Figure 1) [4,8]. In this review we will present the key findings towards the discovery and development of macozinone, an optimized benzothiazinone, which currently appears to be the most advanced DprE1 inhibitor.<smiles>CON(C)O[Na]</smiles>

Figure 1. DprE1 inhibitors in clinical development.

\section{Discovery of Benzothiazinones as Highly Potent Killers of Mycobacterium Tuberculosis}

The very first scientific report on benzothiazinones (BTZs) with extremely high antimycobacterial activity was published in 2009 [9]. These compounds emerged as a result of several years of efforts focused on investigation of a family of ditiocarbamate (DTC) derivatives with antimicrobial properties, which was carried out at the Research Center for Antibiotics in Moscow and the Hans Knöll Institute in Jena [10]. DTCs gained their fame primarily as additives in industry and pesticides, which have been in use since 1930. However, some of them were tested as pharmacological agents for treatment of alcoholism, human immunodeficiency virus (HIV) or cancer [11]. It occurred that selected DTC derivatives had minimal inhibitory concentrations (MICs) between 3-30 $\mu \mathrm{g} / \mathrm{ml}$ for M. tuberculosis, but were active also on several species of fast-growing mycobacteria and multiresistant staphylococci [10]. Thorough structure-activity studies focused on improvement of the activity and selectivity of these compounds for mycobacteria was performed. Selected compounds derived from the basic active ortho nitro-dialkyldithiocarbamate structure showed enhanced activity towards fast growing mycobacteria, with $M$. vaccae being the most sensitive reaching MICs $0.4-1.56 \mu \mathrm{g} / \mathrm{ml}$ [12]. In addition, 3 out of 7 tested molecules were efficient and well tolerated also in the mouse foot pad model of leprosy [12]. Nevertheless, it was clear that reactivity of DTCs poses a significant problem in further development of these compounds despite their activity and rather unexpected low level of toxicity as exemplified by studies in mice [12]. Consequently, a thorough investigation of the metabolic conversion of the most active DTCs containing spiroamine moiety, was undertaken. The drug was incubated with the non-pathogenic strain M. smegmatis and all isolated metabolites were tested for antimycobacterial activity. One of these metabolites was particularly active and its structure established by nuclear magnetic resonance (NMR) revealed the principal BTZ scaffold. A set of novel BTZ derivatives was synthesized and tested against both fast-growing and pathogenic mycobacteria in collaboration with Prof. Stewart Cole at Pasteur Institute in Paris [13]. Surprisingly, these compounds were highly active and selective against mycobacteria in vitro, with MIC values $0.195-1.56 \mu \mathrm{g} / \mathrm{ml}$ for M. tuberculosis, including the resistant strains. The lowest MIC value, $0.023 \mathrm{pg} / \mathrm{ml}$, was obtained for M. fortuitum and 
2,2-[2-methyl-1,4-dioxa-8-azaspiro[4.5]dec-8-yl]-8-nitro- 6-(trifluoromethyl)-4H-1,3-benzothiazin-4-one, later named BTZ038 [9]. The pilot in vivo studies with this compound revealed its potency in the murine model of TB, which was comparable to isoniazid used in the same dose of $25 \mathrm{mg} / \mathrm{kg}$ [13]. These encouraging results warranted further exploration of BTZs as perspective compounds for development of a new TB drug.

\section{Mechanism of Action of Benzothiazinones}

An investigation of mechanism of action of BTZ was carried out within the European Union (EU)-funded consortium New Medicines for Tuberculosis (NM4TB), which was formed in 2006 under the leadership of Prof. Stewart Cole. The compound BTZ038 was initially chosen as a lead molecule. Since it contains a chiral center, pure enantiomers BTZ043 (S) and BTZ044 (R) were prepared and subjected to MIC determination. Both forms showed comparable activities in vitro in M. tuberculosis. Accordingly, only one of them, BTZ043, was subjected to mechanistic studies [9].

Genetic experiments aimed at identification of the BTZ target were carried out by Prof. Giovanna Riccardi and her team at University of Pavia. The two approaches which were chosen for this purpose pointed out to the role of the gene $r 03790$ in conferring resistance against the drug. In the first approach, M. smegmatis was transformed with a cosmid library containing DNA fragments from the same organism. An ortholog of the ro3790 gene from M. smegmatis mc $^{2} 155$, MSMEG_6382, was identified on a cosmid, which resulted in an increase in MICs towards BTZ043. In the second approach, BTZ-resistant strains of M. smegmatis, $M$. bovis BCG and M. tuberculosis were isolated and their resistance was traced to mutations in the same codon in rv3790 and its orthologs. In all mutant strains codons corresponding to Cys387 in ro3790 gene product were replaced by Ser or Gly, which increased the MICs of the resistant strains up to 10,000 times. Accordingly, the mycobacterial species M. avium and M. aurum, in which this position was occupied by Ala or Ser, respectively, were naturally resistant against BTZ, which supported identification of rv3790 as a target [9]. Coincidentally, the function of the protein encoded by this gene was described only a short while before the data on the BTZ target became available. In the course of studies focused on the metabolism of arabinose in mycobacteria carried out by Prof. Michael McNeil with collaborators at Colorado State University in Fort Collins, the rv3790 and rv3791 gene products were proposed to be involved in a unique epimerisation of decaprenylphosphoryl ribose (DPR) to decaprenylphosphoryl arabinose (DPA) [14]. The latter molecule serves as a sole donor of arabinose residues for synthesis of the essential arabinan polymers of the mycobacterial cell wall [15]. It was shown that the conversion takes place via oxidation of the DPR substrate, followed by reduction of the resulting keto-intermediate and thus the two oxidoreductases, rv3790 and rv3791, from the so-called arabinogalactan biosynthesis cluster [16] were ideal candidates to catalyse these reactions. However, at the time it was not possible to conclude which of the two is responsible for oxidation and which for the reduction step. Examination of the effects of BTZ043 on enzymatic conversion of DPR to DPA proved that the compound inhibited the oxidation step. Given the confirmed roles of Rv3790 and Rv3791 in epimerization of DPR, we named them DprE1 and DprE2, respectively (Figure 2) [9].

An important step towards understanding the mechanism of action of BTZs was isolation of a covalent adduct of BTZ043 and DprE1 by Trefzer et al. in 2010 [17]. The authors concluded that reduction of the nitro-group to nitroso derivative is critical for formation of the semimercaptal adduct of BTZ043 and DprE1 through the Cys387, which was verified by mass spectrometry. The following study proved that DprE1 itself is responsible for the reductive activation of DprE1. The electrons needed for the reaction are provided by the enzyme's prosthetic group, FAD, which gets reduced during the catalysis of DPR oxidation (Figure 2) [18]. Accordingly, BTZ represents a typical example of a suicide inhibitor. The proposed mechanism of action was confirmed by crystallographic studies in which structures of DprE1 from M. smegmatis were obtained in native and BTZ043-bound forms. As expected, the semimercaptal adduct was formed only in the presence of the substrate analog, farnesylphosphoryl ribose, which provided electrons for reduction of the FAD cofactor necessary for BTZ activation [19]. 


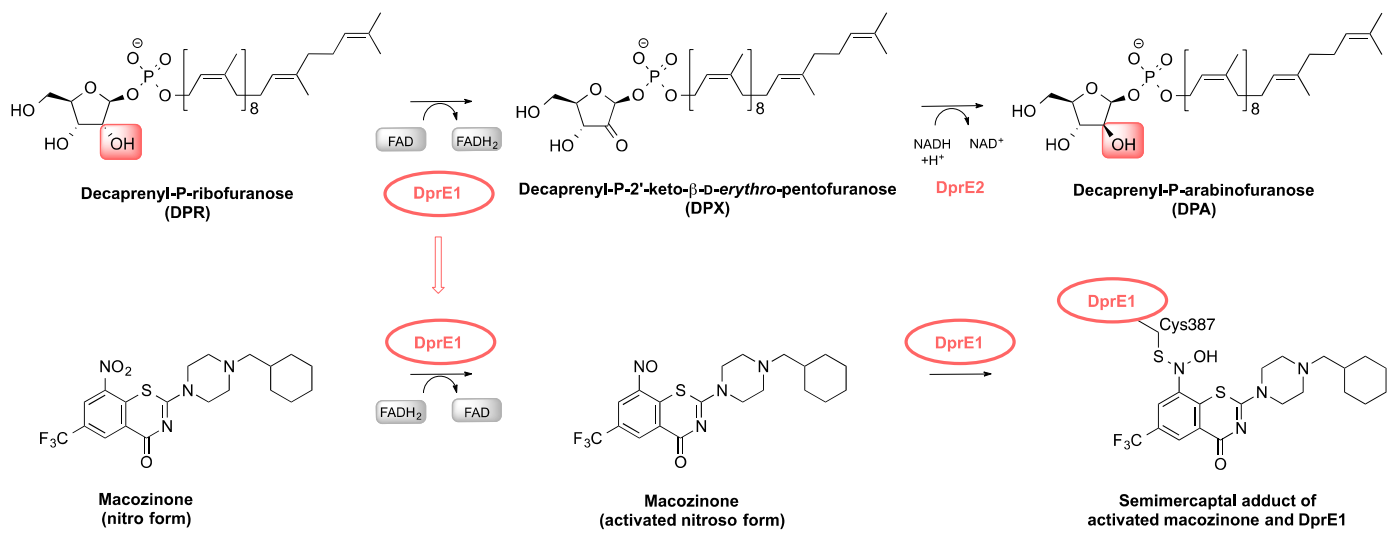

Figure 2. Mechanism of action of macozinone. Upper line: Reactions catalyzed by DprE1 and DprE2. Bottom line: Activation of macozinone and its covalent attachment to DprE1 from M. tuberculosis through Cys387 of the enzyme's active site. The scheme is based on mechanistic studies performed initially with benzothiazinone BTZ043 [17-19] and subsequently confirmed for piperazine-benzothiazinone PBTZ169 (macozinone) [20].

\section{From BTZ043 to PBTZ169}

At the time of its discovery, BTZ043 was the most efficient inhibitor of in vitro mycobacterial growth ever described. While MICs for first line drugs isoniazid or ethambutol for M. tuberculosis were $0.02-0.2 \mu \mathrm{g} / \mathrm{ml}$ or $1-5 \mu \mathrm{g} / \mathrm{ml}$, respectively, the value for BTZ043 was just $1 \mathrm{ng} / \mathrm{ml}$. The compound proved to be active also in intracellular M. tuberculosis infection in Raw 264.7 macrophages and in a mouse model of chronic tuberculosis and it did not show any signs of toxicity or mutagenicity in concentrations well above the amounts used for the experiments. Nevertheless, the extremely low MIC did not translate to comparably high efficiencies in animal studies [9].

In the effort to improve pharmacological properties of benzothiazinone series, structure-activity relationship (SAR) analysis was performed, with main focus on position 2 of BTZ scaffold [20]. It was previously shown that the rest of the positions harbor substituents critical for the antimycobacterial activity, e.g., replacement of 8-nitro-group with any other group, including hydroxylamino-, aminoand nitryl-, led to at least a 500-fold increase in the MIC against $M$. tuberculosis [9]. The new series contained a piperazine at the position 2 of the BTZ structure, which allowed extensive modifications of the N-4 position of this substituent. Evaluation of the 60 prepared piperazine-benzothiazinones (PBTZ) for in vitro activity on M. tuberculosis pointed to a strong correlation between the MICs and lipophilicity. The alkyl-PBTZ derivatives were most active and some of them reached the MICs between 0.19-0.75 $\mathrm{ng} / \mathrm{ml}$ for M. tuberculosis. Five of these derivatives were tested in the chronic TB mouse model, which allowed for selection of PBTZ169 as the most promising candidate [20].

Comparison of BTZ043 with PBTZ169 confirmed superior properties of the latter in all tested parameters. It was three to seven times more active in vitro against $M$. tuberculosis, M. bovis BCG, M. marinum, M. smegmatis, Corynebacterium diphtheriae and C. glutamicum as established by resazurin microtiter assay (REMA). The molecule was also highly active against a panel of 9 MDR- and XDR-clinical isolates of M. tuberculosis [20]. Cross-resistance between BTZ043 and PBTZ169 was confirmed for BTZ-resistant strains of $M$. tuberculosis, M. bovis BCG and M. smegmatis, indicating the common mechanism of action. Interestingly, PBTZ169 was less susceptible to inactivation by nitro-reduction by the nitroreductase NfnB from $M$. smegmatis compared to BTZ043, as confirmed by the liquid chromatography-mass spectrometry analyses. This property could be critical for in vivo activity, due to inevitable actions of host nitroreductases or microbial nitroreductases in the digestive tract of the patients, which would potentially reduce the amount of the available active drug. Enzymologic studies confirmed that PBTZ169 is more efficient inhibitor of its DprE1 target than BTZ043; the enzyme was completely inactivated after 5 min of incubation with $5 \mu \mathrm{M}$ PBTZ169, while four times higher concentration was needed to achieve the same result with BTZ043. The crystal structure of 
M. tuberculosis DprE1 with covalently bound PBTZ169 showed similarities with the BTZ043-bound structure of the M. smegmatis enzyme. However, higher flexibility of the methyl-cyclohexyl group on piperazine, could account for better affinity of this drug for its target and explain its higher capacity to inactivate the enzyme [20].

Efficacies of PBTZ169 and BTZ043 were also evaluated against M. marinum in a zebrafish embryo model. Although after 5 days of treatment both drugs efficiently decreased the bacterial burden at concentrations of $25 \mathrm{nM}$ and $50 \mathrm{nM}$, examination of BTZ043-treated embryos pointed to developmental defects. By contrast, application of PBTZ169 up to $10 \mu \mathrm{M}$ did not cause any pathological changes. In vivo studies in the mouse chronic model of TB proved the superiority of PBTZ169 over BTZ043 in lowering colony forming unit counts in the spleen and lungs, which, however, could not be attributed to better pharmacokinetics, since the two compounds had similar properties except for faster uptake of PBTZ169 [20]. Aiming at the use of the novel PBTZ derivative in designing a new regimen for TB, combination studies with selection of approved and experimental drugs were performed. The combination of PBTZ169 and bedaquiline, which proved to be synergistic in the in vitro REMA assay, was tested in the mouse chronic model of TB. Simultaneous administration of PBTZ, bedaquiline and pyrazinamide was more efficient in reducing the mycobacterial counts both in the lungs and spleen compared to the standard mixture of isoniazid, rifampicin and pyrazinamide [20].

Among favorable properties of PBTZ169 over BTZ043 is the lack of the chiral center, which simplified the production of the drug and significantly decreased its price. This is critical for developing treatment for a disease greatly affecting low-income and middle-income countries in particular. Taken together, PBTZ169 was identified as a promising candidate for development as a novel TB medicine [20].

\section{From PBTZ169 to Macozinone: First Results of the Clinical Studies}

Establishment of a non-profit Innovative Medicines for Tuberculosis Foundation (iM4TB Foundation) at Ecole Polytechnique Fédérale de Lausanne (EPFL) in March 2014 was a principal milestone for the further development of PBTZ169 and its transformation from a valuable TB drug candidate to the compound reaching promising results in the pilot clinical studies (Figure 3) [21]. This approach was necessary to overcome the lack of interest from pharmaceutical companies to address the disease primarily affecting low-income countries. The mission of the foundation is "to develop better and faster-acting medicines to fight tuberculosis and bridge the gap between scientific discovery and the market in order to provide affordable TB treatment to anyone in the world". In July 2014 the foundation joined in this effort with the Russian pharmaceutical company Nearmedic Plus LLC, which bought a license covering the use of PBTZ169 in most countries of the former Soviet Union, while iM4TB retained the rights for the rest of the world. Investments of Nearmedic, along with funds raised by iM4TB, including the generous support of the Bill and Melinda Gates foundation, enabled performing all necessary pre-clinical trials with PBTZ169, such as ADME (absorption, distribution, metabolism, excretion) profiling, toxicology studies, as well as production of clinical supplies of the drug, and preparation of regulatory documents necessary for entering the first clinical trials [21,22].

Clinical studies were initiated by both iM4TB and Nearmedic. In the randomized, double-blind, placebo-controlled Phase 1a study performed in Switzerland (NCT03423030) [23] the aim was to assess safety, tolerability and pharmacokinetic profile of PBTZ169 formulated as a spray-dried dispersion versus a native crystal powder. In addition, antitubercular activity of single escalating doses of PBTZ169 ex vivo was evaluated. The study included 32 healthy male volunteers, grouped to 4 panels of 8 subjects, each undergoing 2 investigation periods, during which they received either single doses of PBTZ169 at increasing dose levels up to $320 \mathrm{mg}$ or a matching placebo. Promising results of this study encouraged the iM4TB team to design of the Phase $1 \mathrm{~b}$ trial (NCT03776500) [24] aimed at evaluation of the safety, tolerability and pharmacokinetics of PBTZ169 in multiple dosing up to $1200 \mathrm{mg} /$ day in healthy volunteers (32 subjects grouped in 4 consecutive panels) receiving PBTZ169 for 14 consecutive 
days. An additional goal is to evaluate interactions of PBTZ16 with the human cytochrome P-450 enzyme family [24].

\begin{tabular}{|c|c|c|c|c|c|}
\hline 2014 & 2015 & 2016 & 2017 & 2018 & $2019 / 2020$ \\
\hline $\begin{array}{l}\text { License agreement } \\
\text { École polytechnique } \\
\text { fédérale de Lausanne } \\
\text { Government contract with } \\
\text { the Ministry of Industry } \\
\text { and Trade of Russia } \\
\text { (MITR) } \\
\text { Start of pre-clinical } \\
\text { studies }\end{array}$ & $\begin{array}{c}\text { Finalizing of pre- } \\
\text { clinical studies }\end{array}$ & $\begin{array}{l}\text { Capsules } \\
\text { of clinical trials (CT) } \\
\text { Government contract } \\
\text { with MITR } \\
\text { for organization GMP } \\
\text { line of substance } \\
\text { synthesis }\end{array}$ & $\begin{array}{l}\text { Start of construction } \\
\text { of substance's } \\
\text { synthesis site }\end{array}$ & $\begin{array}{l}\text { Finalizing } \\
\text { Government contract } \\
\text { with (MITR) }\end{array}$ & $\begin{array}{l}\text { Development \& pre- } \\
\text { clinical studies of new } \\
\text { dosage forms }\end{array}$ \\
\hline
\end{tabular}

Figure 3. Pre-clinical and clinical development of macozinone.

The first Phase 1 clinical trial in Russia (Nearmedic Plus LLC) (NCT03036163, PBTZ169-Z00-C01-1) [25] with an official title "Open-label Prospective Non-comparative Study of Safety, Tolerability and Pharmacokinetics of PBTZ169 after Single and Multiple Fasting Oral Administration in Increasing Doses in Healthy Volunteers" was carried out between January and November 2016. The drug was administered in the form of capsules containing $40 \mathrm{mg}$ of the drug. The study included single escalating doses $(40,80,160,320$, and $640 \mathrm{mg})$ in fasting conditions, as well as multiple fasting doses (320 and $640 \mathrm{mg}$ ) for 14 days. Overall the study included 40 participants [22].

A completed open-label prospective Phase $1 \mathrm{~b}$ study initiated by Nearmedic Plus LLC (NCT04150224, PBTZ169-Z00-C01-3) [26] was designed to assess the safety, tolerability, pharmacokinetics, and food effects on single, double, and multiple escalating doses in healthy volunteers. During the first part of the study, safety, tolerability, and pharmacokinetics of PBTZ169 in $80 \mathrm{mg}$ capsules were studied in healthy volunteers who received single or double (twice daily) fasting doses, which increased sequentially $(640,960$, and $1280 \mathrm{mg}$ once a day and $640 \mathrm{mg}$ twice a day). The food effects were studied for a single $640 \mathrm{mg}$ dose. The second part of the study involved the safety, tolerability, and pharmacokinetics of PBTZ169 in $80 \mathrm{mg}$ capsules in healthy volunteers who received $1280 \mathrm{mg}$ daily in fed conditions, for 14 days. In total, 60 healthy volunteers (10 in each cohort) received the investigational drug and completed the study according to the protocol [22].

During these clinical trials, safety and tolerability of PBTZ169 in capsules were studied, including assessment of adverse effects, vital signs (blood pressure, heart rate, body temperature, respiratory rate), laboratory and instrumental parameters (hematology, blood chemistry, coagulation parameters, urinalysis, electrocardiography) and physical examination. In these studies (NCT03036163 and NCT04150224), good tolerability and favorable safety profile were demonstrated for PBTZ169 in the studied dose range. One event of dose-limiting toxicity (increase in the glucose level 2 hours after the drug administration) was documented after a single $80 \mathrm{mg}$ dose of PBTZ169 in $40 \mathrm{mg}$ capsules (NCT03036163). There were no other cases of dose-limiting toxicity in healthy volunteers in these studies. There were no serious adverse effects. There was no increase in the frequency of adverse effects associated with increased dose or with the administration regimen (fasting or in fed conditions), for single or multiple doses. Changes in some mean vital signs, laboratory, instrumental parameters, or physical examination data were not associated with a trend to increase with a growing dose [22].

Efficacy of PBTZ169 based on the early bactericidal activity (EBA), was studied in a Phase 2a clinical trial (PBTZ169-A15-C2A-1, NCT03334734) [27] by Nearmedic Plus LLC in patients with newly diagnosed smear-positive tuberculosis of the respiratory tract, with preserved sensitivity to isoniazid and rifampicin. Although the trial was terminated early because of slow enrollment, relevant 
information was obtained from 16 patients who participated in the study. The drug was administered in $80 \mathrm{mg}$ capsules, orally as a monotherapy for 14 days, as follows: $160 \mathrm{mg} /$ day dose -4 patients; $320 \mathrm{mg} /$ day dose -4 patients; $640 \mathrm{mg} /$ day dose -7 patients. Isoniazid tablets were used as a control treatment at $600 \mathrm{mg} /$ day dose in one patient.

The primary efficacy of the treatment was evaluated by two methods:

(i) According to quantification of colony forming units in sputum by its inoculation on agar plates, in the group of patients receiving PBTZ169 in the $640 \mathrm{mg}$ dose, EBA 0-14 (mean of the two measurements) and EBA 0-14 (the higher of the two measurements) were $0.071 \log 10 \mathrm{cells} / \mathrm{ml} /$ day [95\% confidence interval (CI) $0 ; 0.143$ ] and $0.080 \log 10 \mathrm{cells} / \mathrm{ml} /$ day $[95 \%$ CI $0.002 ; 0.158]$, respectively.

(ii) According to quantitative polymerase chain reaction, in the same group of patients, EBA 0-14 (mean of the two measurements) and EBA 0-14 (the higher of the two measurements) were $0.098 \log 10$ cells/ml/day [95\% CI 0.021; 0.175] and $0.100 \log 10$ cells/ml/day[95\% CI 0.021; 0.180$]$, respectively.

Thus, the analysis of efficacy based on the data obtained in a pilot Phase 2a clinical study revealed statistically significant early bactericidal activity of PBTZ169 14 days after the start of monotherapy (EBA0-14) in the group of PBTZ169 with the $640 \mathrm{mg}$ dose. During this clinical study, there were no cases of death or severe adverse effects related to the PBTZ169. No adverse effects were considered as definitely related to the drug and, at the study completion, all adverse effects were resolved. There was no increase in adverse effects frequency with growing dose [22].

During the clinical studies it was confirmed that PBTZ169 after single, double, or multiple doses was detectable in the blood plasma of all volunteers and patients who had received the drug. However, it should be noted that pharmacokinetic studies in animals and in healthy volunteers (in Phase 1 clinical studies) demonstrated that PBTZ169, being more soluble in an acidic environment, is absorbed primarily in the stomach. These data agree with the results of in vitro studies, which were conducted by Nearmedic Plus LLC, assessing cellular permeability and solubility in biorelevant media, which mimicked native gastric and intestinal juices. They confirmed that the highest solubility of PBTZ169 is observed at the $\mathrm{pH}$ range of 1 to 2, which coincides with the $\mathrm{pH}$ in the lower part of the stomach, and its solubility gradually decreases with the growing $\mathrm{pH}$. In the media with $\mathrm{pH}$ higher than 5, PBTZ169 was virtually insoluble. According to the test of solubility in biorelevant media, the drug level in the gastric juice was much higher than in the intestinal juice. So, one of the possible methods to increase bioavailability and exposure of PBTZ169 is to prolong its presence in the stomach.

After a single fasting dose, PBTZ169 absorbed rapidly, and the speed of absorption was independent of the administered dose (median $\mathrm{T}_{\max }$ was 1.5-2.5 hours for the dose range 40-640 $\mathrm{mg}$ (NCT03036163), and 1.5-1.75 hours for the dose range 640-1280 mg (NCT04150224). Meantime of retention of the drug in the body was 13.02-19.30 hours and was independent of the administered dose (NCT03036163) [22].

According to the obtained pharmacokinetic data, dose proportionality of $C_{\max }$ and $\mathrm{AUC}_{0-\mathrm{t}}$ was demonstrated for single, as well as for multiple administrations of the investigational drug in the dose range 40-640 mg (NCT03036163, NCT03334734). After single fasting escalating doses (640, 960, and $1280 \mathrm{mg}), \mathrm{C}_{\max }, \mathrm{AUC}_{0-24}, \mathrm{AUC}_{0-\infty}$ were less than dose proportional. Thus, it can be concluded that there is a trend for linear pharmacokinetics for single and multiple doses up to $640 \mathrm{mg}$.

When pharmacokinetics of different administration regimens for $1280 \mathrm{mg}$ daily dose was studied (640 mg twice daily or $1280 \mathrm{mg}$ once in fasting conditions), the pharmacokinetic parameters $\mathrm{AUC}_{0-\infty}$ and $\mathrm{AUC}_{0-\mathrm{t}}$ increased 1.5-1.6-fold (NCT04150224). The relation of mean $\mathrm{AUC}_{0-\infty}$ and $\mathrm{AUC}_{0-\mathrm{t}}$ in cohorts receiving the investigational drug as $640 \mathrm{mg}$ twice a day and $1280 \mathrm{mg}$ once a day, was $153.68 \%$ and $160.32 \%$. For $\mathrm{C}_{\max }$, a slight decrease (1.1-fold) was observed for the $640 \mathrm{mg}$ twice a day (the relation of means was $90.84 \%$ ). All observed differences were statistically significant.

According to the results of analysis of relative bioavailability and relative degree of absorption, the main pharmacokinetic parameters of PBTZ169 substantially and statistically significantly increased when $640 \mathrm{mg}$ dose was administered in fed conditions: $\mathrm{AUC}_{0-\infty}$ increased 3.45-fold, $\mathrm{AUC}_{0-\mathrm{t}}\left(\mathrm{f}^{\prime}\right)$ increased 3.50-fold, $\mathrm{C}_{\max }$ increased 2.29-fold. The drug absorbed and cleared from the body significantly more slowly, when it was administered in fed conditions compared to the fasting conditions (median $\mathrm{T}_{\max }$ 
increased 2-fold, mean $\mathrm{T}_{1 / 2}$ increased 1.44-fold). These data allow to conclude that it is preferable to administer PBTZ169 in fed conditions [22].

In October 2018, the international non-proprietary name "macozinone" was given to PBTZ169 by WHO [28]. In addition to the clinical studies, macozinone is undergoing further in vitro and in vivo testing focused on improvement of its pharmacological properties by structure-based design [29], or on the interactions with anti-TB drugs [30]. Importantly, an efficient method for monitoring metabolism of macozinone in human plasma was recently developed. It enables simultaneous measurement of concentrations of macozinone and its five active metabolites in the clinical samples, which is important for comprehensive pharmacokinetic/pharmacodynamic analyses [31].

\section{Other DprE1 Inhibitors under Active Clinical Development}

Following the discovery of DprE1 as a target of BTZ043, numerous molecules acting on this enzyme emerged, particularly from the whole-cell based phenotypic screening campaigns (for a recent review see [32]). As a result, DprE1 was given an unflattering attribute-a promiscuous target [33]. Nevertheless, DprE1 remains to be one of the best understood and the most vulnerable novel targets. One of the reasons for its high sensitivity against a number of different pharmacophores could be its periplasmic localization, which makes it easily accessible for the drugs [34]. Consequently, in addition to macozinone, there are currently three other DprE1 inhibitors progressing down the clinical studies pipeline. BTZ043, sponsored by the University of Munich; the Hans Knöll Institute, Jena; and the German Center for Infection Research, successfully completed a single dose escalation Phase 1 study (NCT03590600) [35] and is currently recruiting for a combined Phase 1 and 2 study, which will evaluate safety, tolerability, pharmacokinetics and early bactericidal activity of the multiple ascending doses (NCT04044001) [36]. A non-covalent DprE1 inhibitor, TBA-7371 [37] developed by TB Alliance, Bill and Melinda Gates Medical Research Institute and Foundation for Neglected Disease Research, completed a Phase 1 study (NCT03199339) [38] — a partially blind, placebo-controlled study of (i) a combined single ascending dose with a food effect cohort, (ii) multiple ascending dose group, and (iii) a cohort to investigate interactions between TBA-7371 with midazolam and bupropion. Currently, recruitment of adult patients with rifampicin-sensitive pulmonary tuberculosis is taking place for the participation in a Phase 2 study (NCT04176250) [39] to assess safety, early bactericidal activity, and pharmacokinetics of escalating doses of TBA-7371. A combined Phase 1 and 2 study of OPC-167832 by Otsuka is in progress (NCT03678688) [40], aimed at evaluation of the safety, tolerability, pharmacokinetics, and efficacy of multiple oral doses of the drug in patients with uncomplicated drug-sensitive pulmonary tuberculosis with a positive smear. In a parallel group, low dose or high dose OPC-167832 will be combined with delamanid and compared to delamanid only, or standard treatment with isoniazid, rifampin, pyrazinamide, and ethambutol.

\section{Conclusions}

The ultimate goal of the TB drug-development efforts is a design of new, more effective regimen, which could replace the current combination therapy. One of the most promising DprE1 inhibitors is macozinone. Its safety and tolerability profiles, pharmacokinetics, and efficacy in terms of early bactericidal activity were evaluated in the course of three clinical trials conducted by Nearmedic Plus LLC in the Russian Federation, two clinical trials by iM4TB foundation are in progress. High tolerability and a favorable safety profile of the drug in the studied dose range were demonstrated both in healthy volunteers and in patients with newly diagnosed pulmonary tuberculosis with bacterial excretion and preserved sensitivity to isoniazid and rifampicin. The main pharmacokinetics parameters of macozinone after single and multiple administration in the dosage range up to $1280 \mathrm{mg}$ were studied, and a statistically significant efficacy of the drug after monotherapy at a dose of $640 \mathrm{mg}$ a day was established, which allowed the preferred regimen of its intake to be determined.

A thorough study aimed at interactions of macozinone with a spectrum of clinically used and experimental TB drugs was recently performed [30]. It revealed that macozinone does not have 
synergistic or antagonistic interactions with the tested first-line (rifampin, isoniazid, ethambutol) or second-line (amikacin, levofloxacin, moxifloxacin, D-cycloserin, ethionamide, para-aminosalicyc acid) drugs. Among the tested re-purposed or new drugs, clarithromycin, delamanid, lansoprazole sulfide, linezolid, meropenem or sutezolid did not show synergistic effects, when tested individually with macozinone by the checkerboard assay. Synergism was observed for macozinone and clofazimine or bedaquiline, confirming previous findings [20,41]. At the same time, enumeration of colony forming units after drug exposure revealed the next two potentially synergistic candidates-delamanid and sutezolid. In the mouse model of $\mathrm{TB}$, the combination of macozinone-delamanid-sutezolid was more active in the lungs of $M$. tuberculosis-infected animals compared to the regimen of rifampin-isoniazid-pyrazinamide, while the bacterial burden in spleen remained comparable. However, increased toxicity of the combination for HepG2 cells was observed, so further studies are needed to evaluate the potential of this drug combination [30]. Nevertheless, neither antagonism nor increased toxicity was found for most combinations, which paves the way for using macozinone in the development of more efficient TB regimens [30].

Author Contributions: Both authors contributed to writing of the paper. All authors have read and agreed to the published version of the manuscript.

Funding: K.M. acknowledges current support by Slovak Research and Development Agency (APVV-15-0515).

Acknowledgments: Authors would like to thank Nearmedic Plus LLC, and personally to R. Bolgarin for information concerning clinical trials and preclinical studies including unpublished data.

Conflicts of Interest: V.M. is a named inventor on patents pertaining to work on benzothiazinones mentioned herein. K.M. declares no conflict of interest.

\section{References}

1. Bloom, B.R.; Atun, R.; Cohen, T.; Dye, C.; Fraser, H.; Gomez, G.B.; Knight, G.; Murray, M.; Nardell, E.; Rubin, E.; et al. Chapter 11. Tuberculosis. In Major Infectious Diseases. Disease Control Priorities, 3rd ed.; Holmes, K.K., Bertozzi, S., Bloom, B., Jha, P., Eds.; World Bank: Washington, DC, USA, 2017; Volume 6, pp. 233-313.

2. Baer, C.E.; Rubin, E.J.; Sassetti, C.M. New insights into TB physiology suggest untapped therapeutic opportunities. Immunol. Rev. 2015, 264, 327-343. [CrossRef]

3. Global TB Report 2019; WHO: Geneva, Switzerland, 2019; Available online: https://apps.who.int/iris/bitstream/ handle/10665/329368/9789241565714-eng.pdf?ua=1 (accessed on 20 February 2020).

4. Tiberi, S.; du Plessis, N.; Walzl, G.; Vjecha, M.J.; Rao, M.; Ntoumi, F.; Mfinanga, S.; Kapata, N.; Mwaba, P.; McHugh, T.D.; et al. Tuberculosis: Progress and advances in development of new drugs, treatment regimens, and host-directed therapies. Lancet Infect. Dis. 2018, 18, e183-e198. [CrossRef]

5. Andries, K.; Verhasselt, P.; Guillemont, J.; Gohlmann, H.W.; Neefs, J.M.; Winkler, H.; Van Gestel, J.; Timmerman, P.; Zhu, M.; Lee, E.; et al. A diarylquinoline drug active on the ATP synthase of Mycobacterium tuberculosis. Science 2005, 307, 223-227. [CrossRef] [PubMed]

6. Matsumoto, M.; Hashizume, H.; Tomishige, T.; Kawasaki, M.; Tsubouchi, H.; Sasaki, H.; Shimokawa, Y.; Komatsu, M. OPC-67683, a nitro-dihydro-imidazooxazole derivative with promising action against tuberculosis in vitro and in mice. PLoS Med. 2006, 3, e466. [CrossRef] [PubMed]

7. Stover, C.K.; Warrener, P.; VanDevanter, D.R.; Sherman, D.R.; Arain, T.M.; Langhorne, M.H.; Anderson, S.W.; Towell, J.A.; Yuan, Y.; McMurray, D.N.; et al. A small-molecule nitroimidazopyran drug candidate for the treatment of tuberculosis. Nature 2000, 405, 962-966. [CrossRef] [PubMed]

8. WGND Global TB Drug Pipeline. Available online: https://www.newtbdrugs.org/pipeline/clinical (accessed on 20 February 2020).

9. Makarov, V.; Manina, G.; Mikusova, K.; Mollmann, U.; Ryabova, O.; Saint-Joanis, B.; Dhar, N.; Pasca, M.R.; Buroni, S.; Lucarelli, A.P.; et al. Benzothiazinones kill Mycobacterium tuberculosis by blocking arabinan synthesis. Science 2009, 324, 801-804. [CrossRef] [PubMed]

10. Makarov, V.; Moellmann, U. New Dithiocarbamate Derivatives, Useful as Antibacterial Agents, for Treating e.g., Tuberculosis and Infections Caused by Mycobacteria and Staphylococci. U.S. Patent WO2003042186-A, 22 May 2003. 
11. Szolar, O.H. Environmental and pharmaceutical analysis of dithiocarbamates. Anal. Chim. Acta 2007, 582, 191-200. [CrossRef] [PubMed]

12. Makarov, V.; Riabova, O.B.; Yuschenko, A.; Urlyapova, N.; Daudova, A.; Zipfel, P.F.; Mollmann, U. Synthesis and antileprosy activity of some dialkyldithiocarbamates. J.Antimicrob. Chemother. 2006, 57, 1134-1138. [CrossRef]

13. Makarov, V.A.; Cole, S.T.; Moellmann, U. New Benzothiazinone Derivatives Useful for the Treatment of Tuberculosis Infection or Leprosy Infection in Mammals. U.S. Patent WO2007134625-A1, 29 November 2007.

14. Mikusova, K.; Huang, H.; Yagi, T.; Holsters, M.; Vereecke, D.; D’Haeze, W.; Scherman, M.S.; Brennan, P.J.; McNeil, M.R.; Crick, D.C. Decaprenylphosphoryl arabinofuranose, the donor of the D-arabinofuranosyl residues of mycobacterial arabinan, is formed via a two-step epimerization of decaprenylphosphoryl ribose. J. Bacteriol. 2005, 187, 8020-8025. [CrossRef]

15. Wolucka, B.A.; McNeil, M.R.; de Hoffmann, E.; Chojnacki, T.; Brennan, P.J. Recognition of the lipid intermediate for arabinogalactan/arabinomannan biosynthesis and its relation to the mode of action of ethambutol on mycobacteria. J. Biol. Chem. 1994, 269, 23328-23335.

16. Belanger, A.E.; Inamine, J.M. Genetics of Cell Wall Biosythesis. In Molecular Genetics of Mycobacteria; Hatfull, G.F., Jacobs, W.R., Jr., Eds.; ASM Press: Washington, DC, USA, 2000; pp. 191-202.

17. Trefzer, C.; Rengifo-Gonzalez, M.; Hinner, M.J.; Schneider, P.; Makarov, V.; Cole, S.T.; Johnsson, K. Benzothiazinones: Prodrugs that covalently modify the decaprenylphosphoryl-beta-D-ribose 2'-epimerase DprE1 of Mycobacterium tuberculosis. J. Am. Chem. Soc. 2010, 132, 13663-13665. [CrossRef] [PubMed]

18. Trefzer, C.; Skovierova, H.; Buroni, S.; Bobovska, A.; Nenci, S.; Molteni, E.; Pojer, F.; Pasca, M.R.; Makarov, V.; Cole, S.T.; et al. Benzothiazinones are suicide inhibitors of mycobacterial decaprenylphosphoryl-beta-D-ribofuranose 2'-oxidase DprE1. J. Am. Chem. Soc. 2012, 134, 912-915. [CrossRef] [PubMed]

19. Neres, J.; Pojer, F.; Molteni, E.; Chiarelli, L.R.; Dhar, N.; Boy-Rottger, S.; Buroni, S.; Fullam, E.; Degiacomi, G.; Lucarelli, A.P.; et al. Structural basis for benzothiazinone-mediated killing of Mycobacterium tuberculosis. Sci. Transl. Med. 2012, 4, 150ra121. [CrossRef]

20. Makarov, V.; Lechartier, B.; Zhang, M.; Neres, J.; van der Sar, A.M.; Raadsen, S.A.; Hartkoorn, R.C.; Ryabova, O.B.; Vocat, A.; Decosterd, L.A.; et al. Towards a new combination therapy for tuberculosis with next generation benzothiazinones. EMBO Mol. Med. 2014, 6, 372-383. [CrossRef] [PubMed]

21. Innovative Medicines for Tuberculosis. Available online: http://im4tb.org (accessed on 24 February 2020).

22. Mariandyshev, A.O.; Khokhlov, A.L.; Smerdin, S.V.; Shcherbakova, V.S.; Igumnova, O.V.; Ozerova, I.V.; Bolgarina, A.A.; Nikitina, N.A. The main results of clinical trials of the efficacy, safety and pharmacokinetics of the perspective anti-tuberculosis drug Macozinone (PBTZ169). Terapevt. Arkh. 2020, 92, 61-72.

23. Study to Evaluate the Safety, Tolerability, Pharmacokinetics and Ex-Vivo Antitubercular Activity of PBTZ169 Formulation. Available online: https://clinicaltrials.gov/ct2/show/NCT03423030 (accessed on 25 February 2020).

24. Study to Evaluate the Safety, Tolerability and Pharmacokinetics of PBTZ169 in Multiple Dosing. Available online: https://clinicaltrials.gov/ct2/show/NCT03776500 (accessed on 25 February 2020).

25. Phase 1 Study of PBTZ169. Available online: https://clinicaltrials.gov/ct2/show/NCT03036163 (accessed on 25 February 2020).

26. Safety, Tolerability, Pharmacokinetics and Food Effects Study of PBTZ169. Available online: https:// clinicaltrials.gov/ct2/show/study/NCT04150224 (accessed on 25 February 2020).

27. Phase 2a Study of PBTZ169. Available online: https://clinicaltrials.gov/ct2/show/NCT03334734 (accessed on 25 February 2020).

28. WHO Drug Information Volume 32, $\mathrm{N}^{\circ}$ 3. 2018. Available online: https:/www.who.int/medicines/ publications/druginformation/issues/DrugInformation2018_Vol32-3/en/ (accessed on 25 February 2020).

29. Piton, J.; Vocat, A.; Lupien, A.; Foo, C.S.; Riabova, O.; Makarov, V.; Cole, S.T. Structure-Based drug design and characterization of sulfonyl-piperazine benzothiazinone inhibitors of DprE1 from Mycobacterium tuberculosis. Antimicrob. Agents Chemot. 2018, 62. [CrossRef] [PubMed]

30. Lupien, A.; Vocat, A.; Foo, C.S.; Blattes, E.; Gillon, J.Y.; Makarov, V.; Cole, S.T. Optimized background regimen for treatment of active tuberculosis with the next-generation benzothiazinone macozinone (PBTZ169). Antimicrob. Agents Chemot. 2018, 62, e00840-18. [CrossRef] 
31. Spaggiari, D.; Desfontaine, V.; Cruchon, S.; Guinchard, S.; Vocat, A.; Blattes, E.; Pitteloud, J.; Ciullini, L.; Bardinet, C.; Ivanyuk, A.; et al. Development and validation of a multiplex UHPLC-MS/MS method for the determination of the investigational antibiotic against multi-resistant tuberculosis macozinone (PBTZ169) and five active metabolites in human plasma. PLOS ONE 2019, 14, e0217139. [CrossRef]

32. Degiacomi, G.; Belardinelli, J.M.; Pasca, M.R.; De Rossi, E.; Riccardi, G.; Chiarelli, L.R. Promiscuous targets for antitubercular drug discovery: The paradigm of DprE1 and MmpL3. Appl. Sci. 2020, 10, 623. [CrossRef]

33. Lechartier, B.; Rybniker, J.; Zumla, A.; Cole, S.T. Tuberculosis drug discovery in the post-post-genomic era. EMBO Mol. Med. 2014, 6, 158-168. [CrossRef]

34. Brecik, M.; Centarova, I.; Mukherjee, R.; Kolly, G.S.; Huszar, S.; Bobovska, A.; Kilacskova, E.; Mokosova, V.; Svetlikova, Z; Sarkan, M.; et al. DprE1 is a vulnerable tuberculosis drug target due to its cell wall localization. ACS Chem. Biol. 2015, 10, 1631-1636. [CrossRef] [PubMed]

35. A Single Ascending Dose Study of BTZ043. Available online: https://clinicaltrials.gov/ct2/show/NCT03590600 (accessed on 25 February 2020).

36. BTZ-043-Multiple Ascending Dose (MAD) to Evaluate Safety, Tolerability and Early Bactericidal Activity (EBA). Available online: https://clinicaltrials.gov/ct2/show/NCT04044001 (accessed on 25 February 2020).

37. Chatterji, M.; Shandil, R.; Manjunatha, M.R.; Solapure, S.; Ramachandran, V.; Kumar, N.; Saralaya, R.; Panduga, V.; Reddy, J.; Prabhakar, K.R.; et al. 1,4-azaindole, a potential drug candidate for treatment of tuberculosis. Antimicrob. Agents Chemother. 2014, 58, 5325-5331. [CrossRef] [PubMed]

38. A Phase 1 Study to Evaluate Safety, Tolerability, PK, and PK Interactions of TBA-7371. Available online: https://clinicaltrials.gov/ct2/show/NCT03199339 (accessed on 25 February 2020).

39. Early Bactericidal Activity of TBA-7371 in Pulmonary Tuberculosis. Available online: https://clinicaltrials. gov/ct2/show/NCT04176250 (accessed on 25 February 2020).

40. A Phase 1/2 Trial of Multiple oral Doses of OPC-167832 for Uncomplicated Pulmonary Tuberculosis. Available online: https://clinicaltrials.gov/ct2/show/NCT03678688 (accessed on 25 February 2020).

41. Lechartier, B.; Cole, S.T. Mode of action of clofazimine and combination therapy with benzothiazinones against Mycobacterium tuberculosis. Antimicrob. Agents Chemother. 2015, 59, 4457-4463. [CrossRef] [PubMed]

(C) 2020 by the authors. Licensee MDPI, Basel, Switzerland. This article is an open access article distributed under the terms and conditions of the Creative Commons Attribution (CC BY) license (http://creativecommons.org/licenses/by/4.0/). 